\title{
Wireless Affective Brain Computer Interface based Emotion Assessment Model
}

\author{
S. C. Dharmadhikari \\ Pune Institute of Computer \\ Technology, Pune
}

\author{
S.H. Chandak \\ Pune Institute of Computer \\ Technology, Pune
}

\author{
R. R. Chhajed \\ Pune Institute of Computer \\ Technology, Pune
}

\begin{abstract}
Largest proportion of people suffering from depression are women on account of hormonal imbalances occurred owing to varied reasons such as Pregnancy, Polycystic Ovary Syndrome (PCOD), Premenstrual syndrome (PMS), menopause, malnutrition etc. In this respect, it is very much necessary to create awareness among them about drastic implications of depression on their life. Moreover, there is also need to provide them with very easy to use mechanism for detecting and treating depression level at its earlier stage in order to improve the confidence level, decision making and quality of life. In an attempt, this paper address this clinically critical issue by providing valuable comprehensive survey with respect to aforesaid topic and proposing a wireless affective brain computer interaction based easy to use assessment model to detect depression and anxiety levels with the objective to reduce level of depression by contributing to the multidisciplinary fields of neuroscience, psychology and computer science.
\end{abstract}

\section{Keywords}

Affective Brain Computer interface, electroencephalogram, neuroscience

\section{INTRODUCTION}

According to World Health Organization, largest proportion of people suffering from depression are women on account of hormonal imbalances occurred owing to varied reasons such as Pregnancy, Polycystic Ovary Syndrome (PCOD), Premenstrual syndrome (PMS), menopause, malnutrition etc. As in presently, this fact is more evident even in case of working women due to different challenges and impediments that are getting posed in the industries, offices and even in the business in order to sustain in the competition. Extreme work pressure, responsibilities at family front, lack of perseverance attitude and increased expectations in life style induces high level of stress in the women especially in the age group of 2840. Consequently, more depression cases are reported leading to frequent occurrences of emotional imbalances in the form of anxiety, mood swing, lack of confidence, low self esteem along with physical disorders like Acidity, Blood pressure, Diabetes, Heart trouble. Depression is acknowledged as the psychiatric disease and closely related to functional, molecular, and structural changes in the brain. Unfortunately, it has been observed that most of the women are either unaware of or ignoring aforementioned symptoms and are not having willingness to take treatment. Very few, educated women usually take prescription based medication for aforesaid ailments. However, these psychotropic drugs help them feel better and function with more ease, but at the same time put behind the specter of side effects lurking in every bottle of pills. In this respect, it is very much necessary to create awareness among women about drastic implications of depression on their life and other family members too.
Moreover, there is need to provide them with very easy to use mechanism for detecting and treating depression level at its earlier stage in order to improve the confidence level, decision making capacity and quality of life.

In an attempt, this paper address this clinically critical issue by proposing wireless affective brain computer interaction based easy to use assessment model to detect depression and anxiety levels. The field of affective Brain Computer Interface aims to detect affective states of brain such as drastic emotion variations from neuro-physiological signals arose in the form of electroencephalogram wave patterns and utilizes it to enhance human-computer interaction. With this foothold, earlier research attempts made in this field are discussed in the Section 1. The proposed model and its architectural description is presented in Section 2. The probable implications and analysis of the proposed model is provided in Section 3. Important facts, findings and contributions are discussed in Conclusion.

\section{RELATED WORK}

The need and justification for hybrid brain-computer interfacing is highlighted in [1]. It illustrates how the hybrid interfaces may be helpful in the concurrent recording of various physiological signals and elicitation of more than one mental process in order to increase the number of possible input commands and achieve more flexible and robust systems. The proposal aims at systematically reviewing recent articles on the topic of non-invasive hBCIs, to comprehensively identify the current trends, limitations and challenges. Whereas, [2] throws light on use of different music therapy techniques to deal with psycho-affective disorders, social or behavioral difficulties, sensory, physical or neurological disorders. The two types of musical therapies namely receptive music therapy- it is based on music listening and active music therapy - it is the one which gives the person, the possibility to create his own acoustic with the help of musical instrument or with the voice. Furthermore, various application areas that could benefit from brain waves in facilitating or achieving their goals, major usability and technical challenges that face brain signals utilization in various components of BCI system are discussed in [3]. It also provides different solutions that aim to limit and decrease their effects. Then, the technical details and validation of a framework for building multi-platform, portable EEG applications with real-time 3D source reconstruction using wireless EEG sensors with smartphones are observed in [4]. It also narrates the mechanism which combines an off-the shelf neuro headset or EEG cap with a smart phone or tablet, and as such represents the first fully portable system for real time.In [5] pressing clinical need for responsive neuro stimulators, which sense a patient's brain activity and deliver targeted electrical stimulation to suppress unwanted symptoms in psychiatric illness, where symptoms can fluctuate throughout the day is emphasized. The concept and techniques of 
affective BCIs, which decode emotional experience from neural activity, are described empirically. On the similar line, mapping of brain activity using wearable sensors has been discussed in [6]. It demonstrates the process of mapping on a mobile device by connecting the scanner directly to the mobile device over a wireless bluetooth interface. It emphasizes an inherent advantage of this mechanism in providing support to disabled persons in a non medical facility. It alsofacilitates a platform for remote health monitoring of brain related diseases and rehabilitation of patients. The authors of [7] investigates the role of frontal EEG as predictor of clinical response to SSRIs or venlafaxine in major depressive disorder (MDD). It also evaluated prospectively the relative theta power as predictor of treatment outcome and also developed an Antidepressant Treatment Response (ATR) index using EEG parameters assessed at baseline and one week time span. Furthermore, [8] aimed at assessing differences between depressed and normal elderly subjects on alpha asymmetry, and to observe the correlations of this measure with depressive symptoms and quality of life. It concludes that, the relationship between depression and frontal asymmetry was better observed in healthy elderly, where relatively greater left frontal activity was associated with less depressive symptoms.

In [9], the utilization of frontal asymmetry of EEG alpha power (FA) as an index to calculate the risk for anxiety and depression is discussed. Authors examined the heritability of FA in 732 twins and their singleton siblings, and established the genetic and environmental contribution to the relation between FA and the risk for anxiety and depression. Experimentations reveal through Multivariate models that FA is heritable only in young adults (males $32 \%$ and females $37 \%$ ) but not in middle-aged adults. A significant relation between FA and the risk for anxiety and depression was only found in young adult females. Subsequently, authors of [10] have suggested that greater relative right anterior activity may act as a vulnerability marker for the development of depression and anxiety disorders. Through this study they have examined whether anterior and posterior EEG asymmetry patterns predicted anxiety and depressive symptoms one year later. At time 1, participants completed the Beck Depression Inventory (BDI) and State-Trait Anxiety Inventory (STAI) and had baseline EEG activity recorded. Participants then completed the BDI and STAI one year later. Those with relatively greater EEG activity in the right anterior region reported greater trait anxiety one year later. These preliminary results suggest that relative right frontal EEG activity may predict future development of anxiety symptoms.

The authors of [10] emphasizes the recent efforts on Computer Aided Diagnosis of depression using EEG signals with a focus on using nonlinear methods. The importance of early detection of Depression disorder is highlighted in [12] which also throws light on various disorders like sleep disorders and alcoholism caused on account of depression. It demonstrates empirical analysis of Electroencephalogram Gram (EEG) signals obtained from publicly available database using MATLAB. The authors of [13] have focused on mobile phone based applications for physically disabled users, who can't speak or use conventional input device such as keyboard, mouse etc. This paper conducts a survey to give an insight on how to dial a call, compose an SMS (Short Mail Service) and also in Composing of Ringtones using the mobile phone for a BCI system with the help of an EEG based headset. The authors of [14] demonstrated use of J48 algorithm for the classification of EEG based diseases such as: dementia, Schizophrenia, ADHD, epilepsy and mood disorder. Their empirical analysis is shown in terms decision tree, true positive rate, false positive rate and ROC curve. The authors of [15] contributed on remedial side for depression by bringing attention to need of meditation for all human beings in order to tackle challenges and impediments in day to day life. It accentuates the impact of recitation of 'OM' mantra to fetch peace and calm. An author shown demonstration of this fact using wavelet transforms time-frequency analysis and concluded empirically that steadiness in the mind is achieved by chanting $\mathrm{OM}$.

\section{PRPOSED METHODOLOGY AND WORKING OF THE MODEL}

\subsection{Methodology}

The basic scheme of proposed Wireless affective brain computer interaction based emotion assessment model is illustrated in Figure 1. The system consists of four major parts viz. Wireless EEG Signal Acquisition, Real time EEG Signal Processing, Affective State identification and Recommendation system for remedial solution. Wireless EEG Signal Acquisition module will be responsible to record EEG signals simultaneously and then transmit these EEG signals for processing wirelessly. Furthermore, EEG signals will be processed in real-time by storing the relevant estimated EEG features continuously. These EEG signals will be further utilized to identify affective state of Brain and based on its outcome the suitable remedial solution in the form of suggestion for meditation or breathing technique is recommended. Expected functionality of these components is described as below- EEG Signal Acquisition - It mainly consists of amplifier circuit, microprocessor and the Bluetooth transmission circuit. The amplifier circuit is responsible to filter and amplify the EEG signals acquired from the EEG electrode. Then the microprocessor is used to digitize the EEG signal, perform low pass filtering and control the peripheral circuit. It is also responsible to send EEG signals into the Bluetooth transmission circuit. The use of 3-4 electrode based system will be preferred as having many sensors not only increases the cost but also leads to the problem of high dimensional feature space.Real time EEG Signal Processing It will be mainly responsible for preprocessing of acquired signals.Affective State identification - As mentioned in earlier researches, it has been evident that persons with depression have hemispheric asymmetry in their brain signals as compared to normal persons. Identification and analysis of hemispheric symmetry and asymmetry attributes in the form of EEG signals serves the basis of this stage and thus it mainly involves two tasks viz. identification of emotional state and classification of it being stable or unstable/chaotic. Recorded EEG signals are classified based on their frequency - domain correlation as shown in Table-1.Processing of alpha signals has been given more emphasis as earlier studies reported that EEG signal asymmetry at the alpha band in clinically depressed elderly adults are similar to that of younger depressed subjects when compared to normal subjects [11]. Furthermore, [18] reports the use of resting frontal EEG asymmetry as a marker of depression and showed that it is predominant in women. Thus, EEG signal asymmetry at the alpha band and frontal EEG asymmetry has been chosen as parameters to identify and classify affective state. Furthermore, possibly positive cases with inclination towards depressive state have been exposed to recommendation system. It is constituted with questionnaire to measure severity of depression and grade it. Subsequently, as per the grading remedial solution in the form of preliminary 
suggestive helping tutor consisting of music therapy, bija mantra recitation and breathing techniques will be offered.

\subsection{Working}

The proposed application will be presented to user in the form of mobile app and user need to put on wireless BCI head band. Once the application is launched on the mobile device, the app will provide the user with different set of questionnaire/puzzles/quizzes in order to extract the depression/anxiety level. The EEG graph of brain waveforms along with easily comprehendible statistics shall be then presented to user.

If the user's depression/anxiety level is found to be above specified threshold then depending on statistics of variations in Alpha, Beta, Theta and Gama waveform patterns and extracted cause and symptom statistics the associated Chakra may be identified and Bija mantra based guided meditation technique is recommended.

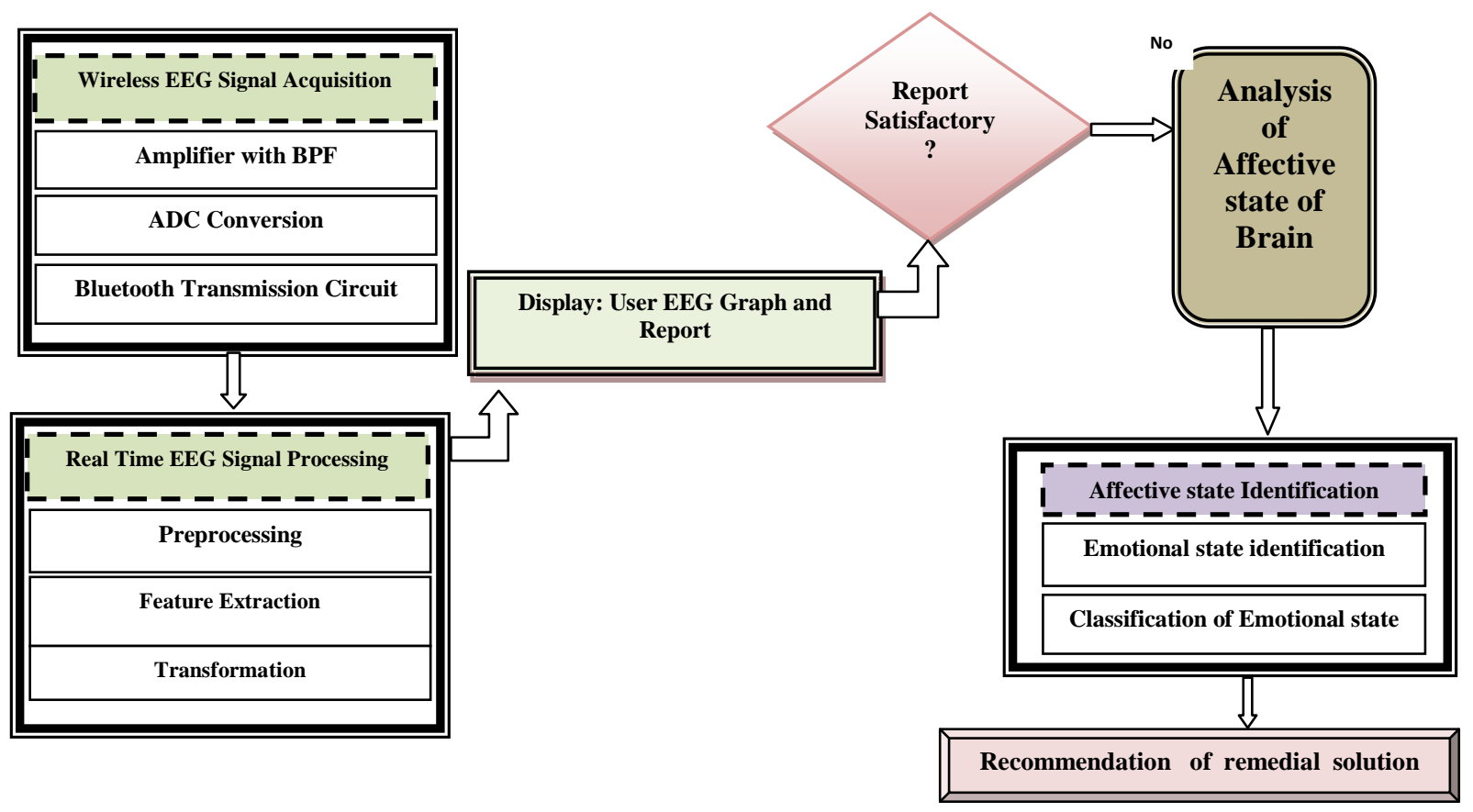

Figure 1: System Architecture of proposed Wireless aBCI based emotion assessment Model

Table 1 : Frequency domain correlation with brain region

\begin{tabular}{|c|c|c|}
\hline Frequency Band & $\begin{array}{c}\text { Frequency range } \\
(\mathrm{In} \mathrm{Hz})\end{array}$ & Associated Brain region \\
\hline delta & $0.5-4$ & Medical Prefrontal cortex, ventral segmental area, nucleus accubens \\
\hline theta & $4-8$ & Limbic and associated structures in the medial prefrontal cortex. \\
\hline alpha & $8-13$ & Parietal region,Occipital region \\
\hline beta & $13-30$ & Sensory-motor system \\
\hline gamma & Above 30 & Sensond non-sensory cortical networks \\
\hline
\end{tabular}

\section{IMPLICATIONS AND ANALYSIS OF THE PRPOSED MODEL}

It has been observed that existing attempts does not taken deeper insight into causes and symptoms towards detecting anxiety and depression which may vary person wise and definitely play important role in deciding plan of action. The proposed work exactly targets this main issue and emphasizes detection of depression and anxiety levels by analyzing cause and symptom correlations ship. Furthermore, it has been observed that emotions and related brain processes have long been neglected in neuroscience which may play important role in early detection of depression, depression which is acknowledged as the psychiatric disease and closely related to functional, molecular, and structural changes in the brain in the form of emotion variations. The proposal addresses this issue by utilizing concept of affective Brain Computer Interface (BCI) in order to detect affective states of brain such as drastic emotion variations from neuro-physiological signals arose in the form of electroencephalogram(EEG) wave patterns. Importantly, most of the earlier BCI based efforts towards detecting depression are based on bulky wired set up which is expensive and not portable and hence not affordable to common man. The proposed model aims at devising 
wireless tool in the form of mobile App which can be freely downloadable and only needs low cost wireless BCI headset.

\section{CONCLUSION}

This paper presents a wireless affective brain computer interaction based easy to use assessment model to detect depression and anxiety levels especially in working women. The objective of proposed model is twofold viz. to create awareness among women and to provide them easy mechanism for dealing with depression caused to high level of stress caused on account of varied reasons such as Pregnancy, Polycystic Ovary Syndrome (PCOD), Premenstrual syndrome (PMS), menopause, malnutrition etc. The paper also provides valuable comprehensive survey with respect to earlier efforts on aforesaid topic. It has been evident from earlier state-of-art that very rare attempts contributes towards investigation and use of Brain Computer interface, electroencephalogram, neuroscience, psychology towards providing technological aid for reinforcement of women.

\section{REFERENCES}

[1] H. Banvillea \&T.H. Falk, “ Recent advances and open challenges in hybrid brain-computer interfacing: a technological review of non-invasive human research," Taylor and Francis Brain-Computer Interfaces, volume 3, Issue 1, pages 9-46, 2016.

[2] Laura D, Sylvie J and Aurore S., "The Effects of Music Therapy on Anxiety and Depression," Annals of Depression and Anxiety- Austin Publishing group. 2015; 2(4): 1057.

[3] Sarah N. Abdulkader, Ayman Atia et. al., "Brain computer interfacing: Applications and challenges," ScienceDirect Egyptian Informatics Journal, Volume 16, Issue 2, Pages 213-230, July 2015

[4] Stopczynski A, Stahlhut C, Larsen JE, Petersen MK, Hansen LK , "The Smartphone Brain Scanner: A Portable Real-Time Neuroimaging System"PLoS ONE 9(2): e86733. doi: 10. 13 71/journal.pone.0086733, Feb 2014

[5] Alik S. Widge, Darin D. Doughertya et. al. , "Affective brain-computer interfaces as enabling technology for responsive psychiatric stimulation," Taylor and Francis Brain-Computer Interfaces, Vol. 1, No. 2, 126-136, 2014.

[6] Syed Muhammad Anwar, Muhammad Majid, and Muhammad Ehatisham-ul-Haq," Mapping Brain Activity Using Wearable EEG Sensors for Mobile Applications," Proceedings of the 2nd International Conference on
Multimedia and Human-Computer Interaction Prague, Czech Republic, August 14-15, 2014 Paper No. 127

[7] Iosifescu DV, Greenwald S, Devlin P, Mischoulon D, Denninger JW, Alpert JE, Fava M: Frontal EEG predictors of treatment outcome in major depressive disorder. Eur Neuro psychopharmacol 2009;19:772-777.

[8] Deslandes AC, de Moraes H, Pompeu FA, Ribeiro P, Cagy M, Capitão C, Alves H, Piedade RA, Laks J: Electroencephalographic frontal asymmetry and depressive symptoms in the elderly. Biol Psychol 2008;79:317-322.

[9] Smit DJ, Posthuma D, Boomsma DI, De Geus EJ: The relation between frontal EEG asymmetry and the risk for anxiety and depression. Biol Psychol 2007;74:26-33.

[10] Blackhart GC, Minnix JA, Kline JP: Can EEG asymmetry patterns predict future development of anxiety and depression? A preliminary study. Biol Psychol 2006;72:46-50.

[11] Acharya U.R., Sudarshan V.K. et. al. , "Computer-Aided Diagnosis of Depression Using EEG Signals," European Neurology,pp.329-336,2015

[12] Deepak R, K R Rachith, "A Survey: Usage of BrainMachine Interface in Various Applications," International Research Journal of Engineering and Technology, vol. 2,Issue- 01, Apr-2015

[13] Mallikarjun HM, Suresh HN: Depression level prediction using EEG signal processing. Contemporary computing and informatics (IC3I). IEEE International Conference, 2014, pp 928-933.

[14] Kambli P, Lingaraju GM, Bhavana S. A study on BCI from the context of Mobile based Application. IJIRAE. 2014 Sep; 8(1). ISSN: 2349-2163.

[15] Deepika Kundra and Babita Pandey Classification of EEG based Diseases using Data Mining. International Journal of Computer Applications 90(18):11-15, March 2014

[16] Ajay Anil Gurjar and Siddharth A. Ladhake, "TimeFrequency Analysis of Chanting Sanskrit Divine Sound "OM" Mantra," IJCSNS International Journal of Computer Science and Network Security, VOL.8 No.8, August 2008.

[17] Deslandes AC, de Moraes $\mathrm{H}$ et. Al. "Electroencephalographic frontal asymmetry and depressive symptoms in the elderly. Biol Psychol 2008, 79, 317-322. 